\title{
Caterine Galaz, Rolando Poblete, Carla Frias. Políticas Públicas e Inmigración ¿̇Posibilidades de Inclusión efectiva en Chile? Santiago: Editorial Universitaria, 2017, 130 págs.
}

Este libro tiene como uno de sus objetivos el dar cuenta y presentar las diferentes miradas sobre la relación del Estado con las personas inmigradas en Chile, acentuando un análisis desde un enfoque de derechos humanos en las políticas públicas sectoriales dirigidas a la población. A fin de ver cómo éstas configuran al "sujeto extranjero" y al "sujeto migrante", de modo tal, de analizarlas relaciones de inclusión/ exclusión social que se viven en los diversos contextos en que los colectivos inmigrados se insertan.

En el primer capítulo, se entrega una contextualización socio histórica y demográfica de los procesos migratorios, puntualizando en los distintos momentos que se han vivido a nivel histórico en Latinoamérica y en especial Chile, y que tienen implicancias para pensar y entender los procesos migratorios de la actualidad. Dentro de esta contextualización, se mencionan las dificultades en la precisión de los datos, no obstante, se entrega un panorama actualizado, integrando distintas fuentes sobre la población inmigrada en Chile. Por otra parte, es importante mencionar que este primer capítulo aporta una caracterización social de los colectivos inmigrados, que da cuenta de los contextos dinámicos en que estos se insertan y las vulnerabilidades que desde allí emergen. Cuestión que los sitúa en una posición de desventaja en relación a la población chilena y que agudiza la relevancia de acciones que impliquen un rol activo desde el Estado. Tal como plantea Heras[1], han sido actores de la sociedad civil quienes han debido reemplazar y complementar acciones que son responsabilidad del Estado.

El segundo capítulo invita a una discusión y estudio sobre el concepto de inclusión y su complejidad teórica - operacional, desde una perspectiva de derechos humanos. Sin duda, el rol del estado cobra relevancia en las definiciones que establece desde sus políticas y legislaciones sobre conceptos como el de la inclusión, situándolo en un contexto socio cultural diverso y dinámico que se sostiene en la base de exclusiones y desigualdades históricas. Desde lo anterior, este segundo capítulo abre un espacio de discusión, al plantear la construcción de nuevas fracturas sociales y subjetividades que configuran las desigualdades y vulneran la inclusión como derecho humano.

En el tercer capítulo, se presentan experiencias internacionales de países como España, Alemania, Argentina, Costa Rica, Uruguay y Ecuador, lo que

Caterine Galaz, Rolando Poblete, Carla Frias. Políticas Públicas e Inmigración

¿Posibilidades de Inclusión efectiva en Chile? Santiago: Editorial Universitaria, 2017, 130 págs.

Valeria Acuña Ramírez

Autoctonía. Revista de Ciencias Sociales e Historia, Vol. II, N¹, Enero-Junio 2018, 163-166

ISSN 0719-8213

DOI: http://dx.doi.org/10.23854/autoc.v2i1.61 
permite realizar una comparación y también nutrir la observación de las políticas en Chile a modo de aprendizaje. El capítulo logra sintetizar de manera acabada una contextualización de los países ya mencionados y sus experiencias en cuanto a políticas migratorias y políticas sociales inclusivas, haciendo un contraste con el enfoque de derechos humanos. Por otra parte, este tercer capítulo abre un camino de discusión figurando la idea de esperanza, en cuanto a observar la voluntad de los gobiernos latinoamericanos para establecer normativas legales que garanticen el respeto de los derechos humanos. También es importante reconocer la dinámica lenta de integración regional que permita, tal como plantea el texto, "desnacionalizar" las políticas al existir espacios regionales en común. Lo anterior abre puertas al lector desde cual sea su espacio de acción a pensar e indagar en puentes dialógicos que encaminen a una participación que incorpore a todos los sectores de las distintas regiones, y es importante considerar al pensar en una integración entre las distintas regiones de Latinoamérica en aspectos históricos señalados por Novick[2], quien plantea que desde la emancipación colonial, la integración regional fue un objetivo negado y buscado.

En el capítulo cuatro, se plantea el rol de las políticas públicas en materia de migración. Allí surge una mirada crítica al concepto de "integración" siguiendo de cerca a Foucault [3]- que dice relación con pensar las políticas públicas como dispositivos sociales ligados a la normatividad. Por otra parte, este cuarto capítulo entrega una síntesis acabada de las convenciones internacionales vigentes en Chile, que tienen relación con los derechos sociales de los/as inmigrantes, también los ejes y principios de la Política Nacional Migratoria en Chile desde una mirada crítica y reflexiva. Siempre teniendo como eje un enfoque de derechos humanos cuyo análisis se considera un aporte para la construcción de políticas públicas que tengan como fin la inclusión.

Por último, se mira el rol que cumplen los/as funcionarios/as de los diferentes aparatos públicos y estatales en la producción y reproducción de las políticas públicas. Así, se plantea la subjetividad en la transformación y recreación de las políticas y sus procedimientos. Lo anterior, invita a pensar aspectos de la inclusión y la relación entre las personas y cómo dichas relaciones se configuran en las subjetividades y los contextos, pero también desde el accionar estatal. En cuanto a esto, se puede mencionar lo planteado por Stefoni[4] sobre la política de la no política, que tiene que ver con el acceso a los derechos y quedaría sujeto a disposición de los funcionarios y ciudadanos que estarían en posiciones de poder frente a los inmigrantes. Así, Stefoni plantea la idea de la ausencia de políticas que, en este libro, en particular, se toma como una idea que forma parte de la subjetividad propia de los funcionarios, aun habiendo normativas que puedan ser inclusivas, éstas no se llevan a la práctica como tales.

La segmentación que surge durante la dictadura y el temor a la otredad, son una verdadera fractura existente en la sociedad chilena. Por lo tanto, se valora que en este capítulo, se presente un estudio que otorga un componente histórico, porque es fundamental al considerar las subjetividades construidas en los funcionarios públicos que ejecutan las políticas.

El capítulo da cuenta de algunos resultados de una investigación en la que participan los/as autores/as del libro acerca de la oferta pública, participación, inclusión y vulnerabilidad de la población migrante en Chile. Lo anterior, en cuatro sectores: educación, salud, vivienda y trabajo.

Destaca que este capítulo otorga una mirada crítica desde los obstaculizadores a los diferentes recursos, 
así como también los facilitadores, agradeciendo los aspectos recursivos que invitan a fortalecer y mejorar aquellas prácticas y lineamientos que apunten hacia la inclusión. Por otra parte, se plantean las barreras procedimentales y culturales que dificultan el acceso a los procedimientos, servicios y políticas. En este aspecto, es importante mencionar que el capítulo brinda relatos desde actores de distintos espacios sociales, lo que enriquece el análisis y reflexiones del texto.

Por último, las/os autoras/es en sus reflexiones finales enfatizan la importancia de la perspectiva de los derechos humanos, no solo como marco ético, sino también procedimental y constructor de relaciones, en un país que aún no logra asegurar estos derechos a los inmigrados. Según los autores, aún no pueden situarse como sujetos ciudadanos que puedan ejercer esta potestad, lo que nos sitúa en la emergencia de generar acciones, debates y reflexiones entre los distintos espacios y sectores de la sociedad que apunten a la construcción de una sociedad inclusiva que considere la diversidad socio cultural.

Se valora que el libro presenta una contextualización social, demográfica y política desde las legislaciones y servicios básicos de manera acabada y sintetizada. Desde ahí, es que se convierte en un material importante de lectura y estudio, para contar con una panorámica completa de la situación actual. Permite poder instalar un debate sobre la inclusión, la construcción de ciudadanía, los espacios de participación en un escenario de movilidad humana y de diversidad socio cultural que se construye en bases de desigualdad social en que los colectivos inmigrados están en una posición de desventaja y vulnerabilidad.

Como una crítica general, se puede señalar que el libro no profundiza en colectivos tales como la infancia o las mujeres migrantes, si bien menciona que son grupos que están teniendo gran presencia en el país, no se hace alusión a políticas de niñez y de género, que se consideran importantes al pensar en la inclusión desde un enfoque de derechos humanos. Los estudios sobre las situaciones de los inmigrantes dan cuenta de que en temáticas de género, trabajo y niñez, existe alta evidencia de discriminación, lo que justifica la necesidad de incorporar en este análisis desde las políticas públicas el componente de niñez y género[5].

Además, si bien el libro invita a reflexionar sobre la complejidad en que se configuran las relaciones humanas y la importancia que tiene el Estado y las miradas que propone sobre los sujetos, puesto que dichas miradas supondrán también formas de relacionarse y que están en interacción con las subjetividades propias de las personas y espacios sociales; se echa en falta una mirada hacia las políticas públicas desde diferentes actores sociales y territorios de los diferentes sectores, a fin de conocer cómo se están configurando las colectividades y sus subjetividades y propuestas en términos de participación y ciudadanía.

\section{NOTAS}

[1] Heras, María José, "Los migrantes como sujetos del sistema de protección social en Chile". Temas de la Agenda Pública Universidad Católica. Año 11, $\mathrm{n}^{\mathrm{o}}$ 91, 2016. [En línea] Recuperado de: http://politicaspublicas.uc.cl/ wp-content/uploads/2016/12/N\%C2\%B0-91Migrantes-como-sujeto-de-protecci\%C3\%B3nsocial.pdf 
[2] Novick S. "Migraciones, políticas e integración regional.: avances y desafíos". [En línea] Recuperada de: http://webiigg.sociales. uba.ar/pobmigra/introdnovick_2010.pdf

[3] Foucault, Michel. Seguridad, Territorio, Población. México: Fondo de Cultura Económica, 1977.

[4] Stefoni, Carolina. "Ley y política migratoria en Chile: la ambivalencia en la comprensión del migrante", en: B. Feldman Bianco (comp.), La construcción social del sujeto migrante en América Latina: prácticas, representaciones y categorías. Quito/Santiago: CLACSO, FLACSO, Universidad Alberto Hurtado.
[5] Pavez, Iskra. Inmigración y racismo: Experiencias de niñez peruana en Santiago de Chile. Si Somos Americanos 12-1 (2012): 75-99.
Valeria Acuña Ramírez Universidad Bernardo O'Higgins, Chile 